Pacific Journal of Mathematics

EXISTENCE OF A CLASS OF STEADY PLANE GRAVITY 


\title{
EXISTENCE OF A CLASS OF STEADY PLANE GRAVITY FLOWS
}

\author{
D. S. CARTER
}

1. Introduction. A number of exact solutions representing free boundary flows of an incompressible fluid under gravity appear in the literature (see [1] and references given there). As pointed out in [1], however, these are obtained by "inverse" methods, and exact theoretical treatment of problems in the large, having prescribed fixed boundaries and singularities, appears to have been confined to the case of periodic gravity waves.

In this paper we consider the family of steady plane irrotational flows of an incompressible inviscid fluid in a uniform gravitational field, with geometric configurations as illustrated in Figure 1. The fluid is

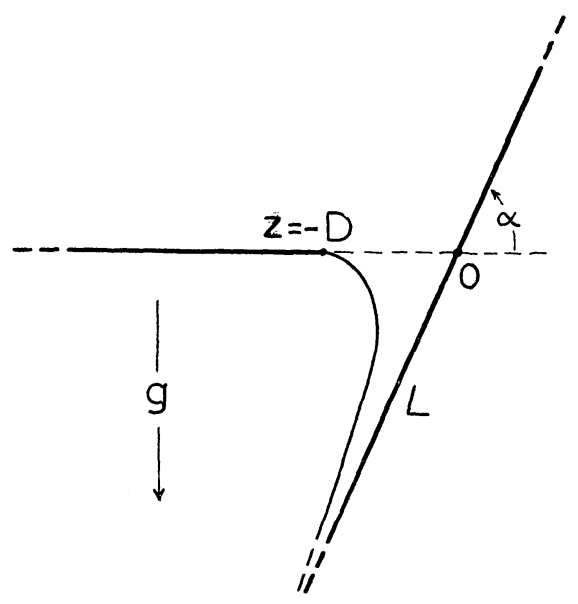

supported by a semi-infinite horizontal plane, and is bounded on the right by an infinite plane inclined at an angle $\alpha$ with the horizontal. The flow is downward through an open slot in the horizontal plane into a jet with a free boundary extending to infinity. This family includes the case of the symmetric jet from a slot, obtained when $\alpha=\pi / 2$ by reflecting the flow across the vertical boundary.

In addition to the angle $\alpha$, the physical parameters entering the problem include the constant specific force of gravity $g$, the slot width $D$, the fluid velocity at the slot edge $q$, and the total flow rate $A$ (crosssectional area of fluid entering the jet per unit time). Our principal result, contained in Theorem $1, \S 3$, and Theorem $4, \S 11$, is that

Received June 15, 1960. Work done partially under the auspices of U.S. Atomic Energy Commission at University of California, Los Alamos Scientific Laboratory, and during tenure of a Temporary Membership, Institute of Mathematical Sciences, New York University. 
there is a similarity class of such flows for each choice of the dimensionless pair $\alpha, g A / q^{3}$, such that $0<\alpha<\pi, g A / q^{3}>0$. (Questions of uniqueness, continuity and monotonicity of the solutions will be discussed in another communication, where it will be shown that each pair $\alpha, g A / q^{3}$ determines a unique similarity class).

A noteworthy property of the solutions is the convexity of the free boundaries. It is implicit in the method of proof that the inclination of each free boundary arc decreases monotonically from zero at the slot edge to $-\pi+\alpha$ as an asymptotic limit.

The problem is formulated in $\S 2$, and reduced in $\S 3$ to a nonlinear boundary value problem on the unit disk in an auxiliary complex plane ( $t$-plane), involving the parameters $\beta=2(\pi-\alpha) / \pi, \lambda=6 g A / \pi q^{3}$, and the function $u=(w / q)^{1 / \beta}$, where $w$ is the conjugate flow velocity. To circumvent a singularity due to to the infinite nature of the free boundary, a modified problem is introduced in $\S 4$, with solutions corresponding to flows as illustrated in Figure 2, where the free jet extends only a finite

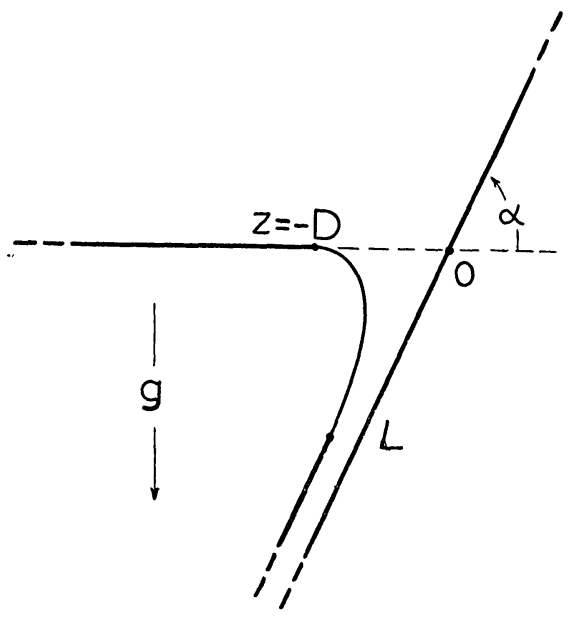

distance before flowing into a straight channel. The existence of these finite jet solutions (Theorem $2, \S 4$ ) is established in the next five sections by a combination of fixed point and conformal mapping techniques. In $\S 10$ the analytic continuation of finite jet solutions beyond the unit disk is discussed, in preparation for the proof in $\S 11$ that the infinite jet solutions exist as limits of normal families of finite jet solutions.

2. Mathematical formulation. Position in the complex plane of the flow is denoted by $z=x+i y$, the complex velocity potential by $W=U+i V$, and the conjugate velocity by $w=d W / d z$. For brevity, the line $\mathfrak{I} \mathfrak{m}\left\{e^{-i \alpha} z\right\}=0$ is denoted by $L$. Then the mathematical construction corresponding to the flow consists of

(a) a simply-connected domain in the $z$-plane, bounded on the right 
by $L$, and bounded below and on the left by a simple curve consisting of a negative real segment $-\infty<z \leqq-D$ and a free boundary arc extending downward to $y=-\infty$.

(b) a function $W(z)$ which maps this domain conformally onto the strip $0<V<A$ so that the horizontal boundary and the free boundary go into $V=0$, and the inclined boundary $L$ goes into $V=A$. The derivative $w=d W / d z$ is continuous on the closure of the flow domain, has the positive value $w(-D)=q$ at the slot edge, and satisfies the condition of constant pressure

$$
|w|^{2}=q^{2}-2 g y
$$

on the free boundary.

The solutions established below have the additional property

(c) The inclination of the free boundary is strictly decreasing from the value zero at the slot edge to the asymptotic limit $-\pi+\alpha$ near $y=-\infty$ where the free boundary is asymptotic to $L$.

3. Reduction to a problem on the unit disk. Following a standard hodograph method (outlined in [1]), the problem can be reduced to a nonlinear boundary value problem on the unit disk in an auxiliary plane. For purposes of the existence proof we reverse the usual derivation, and start with a direct formulation of the reduced problem:

Let $\beta$ and $\lambda$ be real numbers such that $0<\beta<2, \lambda>0$. A function $u(t)$ is a solution of the reduced problem corresponding to the pair $\beta, \lambda$, provided $u$ is

(A) continuous on the unit disk $|t| \leqq 1$, with $t=i$ and $t=-i$ excluded, and regular and univalent on the interior,

(B) real on the real diameter and pure imaginary on the imaginary diameter,

(C) a solution of the following integral equation on the quartercircle $t=e^{i \sigma}, 0 \leqq \sigma<\pi / 2$ :

The modulus $v(\sigma)=\left|u\left(e^{i \sigma}\right)\right|$ and principal argument $\varphi(\sigma)=\arg u\left(e^{i \sigma}\right)$ are related by

$$
v(\sigma)=\left\{1+\lambda \int_{0}^{\sigma} \tan \rho \sin [\beta \varphi(\rho)] d \rho\right\}^{1 / 3 \beta}
$$

(D) monotonic on the same quarter-circle in the sense that as $\sigma$ varies from 0 to $\pi / 2, \varphi$ is strictly increasing between the same limits, and $v(\sigma)$ is also strictly increasing.

To interpret the reduced problem we have

Theorem 1. Let $\alpha, g, q, A$ be strictly positive numbers, where $\alpha<\pi$. Let $u(t)$ be a solution of the reduced problem corresponding to the pair $\beta=2(\pi-\alpha) / \pi, \lambda=6 g A / \pi q^{3}$. Let 


$$
\begin{aligned}
W(t) & =(2 A / \pi) \log \left[t /\left(1+t^{2}\right)\right] \\
w(t) & =q u^{\beta}(t) \\
z(t) & =\int^{t}[1 / w(s)] d W(s) .
\end{aligned}
$$

Then the constant of integration can be chosen so that $z(t)$ maps the first quadrant of the unit disk onto a flow domain as described above. The positive real radius goes into the horizontal part of the flow boundary, the positive imaginary radius goes into $L$, and the circumference goes into the free boundary. The function $W$, "transplanted" to the flow plane by the mapping $z(t)$, is the complex potential for a flow of the required type with the given flow parameters; and the function $w$, similarly transplanted, is the conjugate velocity.

The proof is contained in the following five propositions:

1. The function $w(t)$ maps the first quadrant of the unit disk into the sector $0 \leqq \arg w \leqq \pi-\alpha$ so that $w(1)=q$, the real radius goes into $\arg w=0$, and the imaginary radius goes into arg $w=\pi-\alpha$. The circumference goes into a monotonic arc in the sense that $\arg w\left(e^{i \sigma}\right)$ is strictly increasing between the limits $0, \pi-\alpha$, and $\left|w\left(e^{i \sigma}\right)\right|$ is strictly increasing. Moreover $w$ has an expansion of the form $t^{\beta} \sum_{n=0}^{\infty} a_{2 n} t^{2 n}$ with real coefficients, and with $a_{0}>0$, convergent for $|t|<1$.

Proof. Conditions (C) and (D) on $u$ imply $u(1)=v(0) e^{i \varphi(0)}=1$. Since $u$ is univalent, symmetric about the real and imaginary axes in the sense of (B), and positive at $t=1$, the real and imaginary parts of $u$ agree in sign with those of $t$. Therefore the Maclauren expansion of $u$ has the form $t\left(b_{0}+b_{2} t^{2}+\cdots\right)$, in odd powers of $t$ with real coefficients, and with $b_{0}>0$. The proposition follows with the help of condition (D), since $w=q u^{\beta}, \beta=2(\pi-\alpha) / \pi$.

2. The constant of integration can be chosen so that $z(t)$ has an expansion of the form $t^{-\beta}\left(c_{0}+c_{1} t^{2}+\cdots\right)$ with real coefficients and with $c_{0}<0$, convergent for $|t|<1$. Thus $z(t)$ maps the positive real radius onto a real segment extending to $x=-\infty$, and the positive imaginary radius goes onto a segment of $L$ extending to $y=+\infty$.

Proof. From equations (3) and (4) we obtain

$$
d z / d t=(1 / w)(d W / d t)=2 A\left(1-t^{2}\right) /\left[t\left(1+t^{2}\right) \pi w\right] .
$$

In view of 1 , therefore, $d z / d t$ has an expansion $t^{-1-\beta}\left[d_{0}+d_{2} t^{2}+\cdots\right]$ with real coefficients and $d_{0}>0$. The proposition follows on taking the termby-term integral $z=t^{-\beta}\left[-d_{0} / \beta+d_{2} t^{2} /(2-\beta)+\cdots\right]$.

Henceforth the integration constant is determined as in 2 , and the 
branch of the logarithm in equation (3) is chosen so that

3. The function $W(t)$ maps the first quadrant onto the strip $0 \leqq$ $V \leqq A$, so that the real radius and circumference go into $V=0$ and the imaginary radius into $V=A$.

4. The free boundary equation (1) is satisfied on the curve $z\left(e^{i \sigma}\right)$, $0 \leqq \sigma<\pi / 2$.

Proof. Equations (2) and (4) imply

$$
\left|w\left(e^{i \sigma}\right)\right|^{3}=q^{3}\left\{1+\lambda \int_{0}^{\sigma} \tan \rho \sin \left[\arg w\left(e^{i \rho}\right)\right] d \rho\right\} .
$$

Setting $\lambda=6 g A / \pi q^{3}$, differentiating, and dividing by $3|w|$ gives $|w| d|w|=$ $\left[2 g A \tan \sigma_{\Im} \mathfrak{m}\{w\} / \pi|w|^{2}\right] d \sigma$. Here

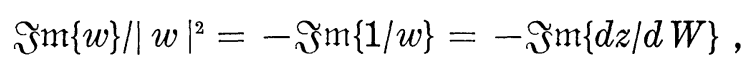

and in view of (3), $d W=(2 A / \pi) \tan \sigma d \sigma$. Hence $|w| d|w|=-g \mathfrak{\Im m}\{d z\}=$ $-g d y$. Integrating gives (1), since $|w(1)|=q$.

5. The function $z(t)$ maps the first quadrant onto a flow domain as described above.

Proof. First, the free boundary arc $z\left(e^{i \sigma}\right)$ extends to $y=-\infty$. This follows from (1) provided $w\left(e^{i \sigma}\right)$ is unbounded. From the last paragraph we have $|w| d|w|=\left[\left.2 g \mathfrak{\Im m}\{w\}|| w\right|^{2}\right\} d W$. In view of $1, \mathfrak{\Im m}\{w\}$ is positive and bounded away from zero near $\sigma=\pi / 2$. Therefore $|w|^{4}$ exceeds a positive constant times $W$, which is unbounded.

Secondly, the free boundary is convex in the sense that its inclination $\arg \{d z / d W\}=-\arg w\left(e^{i \sigma}\right)$ is decreasing between the limits $0, \alpha-\pi$, in accordance with 1.

Now let $\boldsymbol{U}$ denote the image under $z(t)$ of a curve $U=\operatorname{Re}\{W(t)\}=$ constant, directed from the positive imaginary radius to the circumference near $t=i$. From (5) we have $d\left(e^{-i \alpha} z\right)=\left(i \mid e^{i \alpha} w\right) d V$ on $\boldsymbol{U}$. Here $V$ is decreasing, and in view of $1 \operatorname{Re}\left\{e^{i \alpha} w\right\}<0, \mathfrak{\Im} m\left\{e^{i \alpha} w\right\}>0$, so that $\operatorname{Re}\left\{e^{-i \alpha} z\right\}$ is decreasing and $\mathfrak{I} \mathfrak{m}\left\{e^{-i \alpha} z\right\}$ is increasing on $\boldsymbol{U}$. According to 2 the initial point of $\boldsymbol{U}$ is on $L$. The other endpoint is on the free boundary; and it follows in view of the convexity that the entire free boundary curve is in the half-plane $\mathfrak{F}\left\{\left\{e^{-i \alpha} z\right\}>0\right.$, on the left of $L$. Moreover $\boldsymbol{U}$ does not cross either $L$ or the free boundary. Finally, the length of $U$, $\int_{0}^{A}|w|^{-1} d V$, vanishes as $U \rightarrow+\infty$ (i.e., as the curve $U=$ constant "approaches" $t=i$ ); for we have observed that $\left|w\left(e^{i \sigma}\right)\right|$ is unbounded near $\sigma=\pi / 2$, so that $|w|^{-1}$ vanishes near $t=i$. 
Together with 2 , these results show that $z(t)$ maps the real radius and circumference onto the left boundary of a flow domain as described in $\S 2$, condition $(a)$, that the imaginary radius goes into the entire line $L$, and that the free boundary is asymptotic to $L$. To show that the interior of the first quadrant goes into the domain between these two boundaries, consider the image $U^{\prime}$ of a second curve $U=$ constant joining the real and imaginary radii near $t=0$. From the expansion of $z(t)$ in 2 we see that $U^{\prime}$ joins the negative real axis with $L$, without crossing either. By a familiar application of the argument principle it follows that $z(t)$ is univalent and takes its values in the required domain.

Theorem 1 now follows, since composition of $W(t)$ with the inverse of $z(t)$ is the complex potential of a flow with conjugate velocity $w=$ $d W / d z$.

4. The finite jet approximation. To avoid the singularity in the integral equation (2) due to the unbounded factor $\tan \rho$, we will replace $\tan \sigma$ by the bounded function

$$
h_{\gamma}(\sigma)=2 \sin 2 \sigma /\left(2 \cos 2 \sigma+\gamma^{2}+\gamma^{-2}\right), \quad 0<\gamma<1
$$

which approximates $\tan \sigma$ when $\gamma$ is near 1 . More precisely, we will consider the "finite jet problem" obtained by modifying the boundary value problem of $\S 3$ as follows: First, $u$ is required to be continuous for all $|t| \leqq 1$, and secondly, equation (2) is replaced by

$$
v(\sigma)=\left\{1+\lambda \int_{0}^{\sigma} h_{\gamma}(\rho) \sin [\beta \varphi(\rho)] d \rho\right\}^{1 / 3 \beta} .
$$

An appropriate modification of Theorem 1, in which $W(t)$ is replaced by $W_{\gamma}(t)=(A / \pi) \log \left[t^{2}\left(t^{2}+\gamma^{2}\right)^{-1}\left(t^{2}+\gamma^{-2}\right)^{-1}\right]$, shows that solutions of this problem provide flows as illustrated in Figure 2. In the next five sections we will prove

THEOREM 2. The finite jet problem has a solution for each triple $\beta, \gamma, \lambda$, such that $0<\beta<2,0<\gamma<1$, and $\lambda>0$.

5. The operator $T$. Let $\Phi$ be the family of all continuous realvalued functions $\varphi(\sigma)$ on the interval $[0, \pi / 2]$, which are nondecreasing between the limits $\varphi(0)=0, \varphi(\pi / 2)=\pi / 2$, and such that $\varphi(\sigma) \leqq \sigma$. Now we define an operator $T$ on $\Phi$ which serves to reduce the finite jet approximation to a fixed point problem:

First, with each $\phi \in \Phi$ we associate the complex-valued function $v e^{i \varphi}$, where $v(\sigma)$ is determined by $\rho$ through equation (7). Now the integrand in (7) vanishes wherever $\varphi(\rho)=0$, but is strictly positive as soon as $\varphi(\rho)$ becomes positive. Hence $v=1$ where $\varphi=0$, and $v$ is 
strictly increasing thereafter. Moreover $v$ is bounded independently of $\varphi$ by the number

$$
M=\left[1+\lambda \int_{0}^{\pi / 2} h_{\gamma}(\rho) d \rho\right]^{1 / 3 \beta}
$$

Combining this with the requirement $\mathcal{P}(\sigma) \leqq \sigma$, we have

Lemma 1. For each $\varphi \in \Phi$ the function ve $e^{i \varphi}$ maps $[0, \pi / 2]$ onto $a$ simple Jordan arc $C_{I}$ in the u-plane, in the intersection of the first quadrant with the annulus $1 \leqq|u| \leqq M . \quad C_{I}$ intersects the real and imaginary axes only in its endpoints $u=1$, iv( $\pi / 2)$. Moreover this mapping is a homeomorphism of $\left[\sigma_{0}, \pi / 2\right]$ onto $C_{I}$, where $\sigma_{0}=$ $\sup \{\sigma: \varphi(\sigma)=0\}$.

Next we adjoin to $C_{I}$ its reflections about the real and imaginary axes and through the origin. This provides a simple closed Jordan curve $C$, which encloses a simply-connected domain $D$, symmetric about both axes and containing the origin. Let $\hat{u}(t)$ be the function which is continuous for $|t| \leqq 1$ and which maps $|t|<1$ conformally onto $D$ so that $\widehat{u}(0)=0$ and the derivative $\hat{u}^{\prime}(0)$ is positive. Let $\hat{v}(\sigma)=\left|\widehat{u}\left(e^{i \sigma}\right)\right|$ and $\widehat{\varphi}(\sigma)=\arg \hat{u}\left(e^{i \sigma}\right)$ for $0 \leqq \sigma \leqq \pi / 2$. The operator $T$ is defined by $T \varphi=\hat{\varphi}$.

Leaving aside for the moment the question of whether $T$ does in fact map $\Phi$ into itself, we have

Lemma 2. Suppose that $T$ has a "fixed point" $P$ which is also a strictly increasing member of $\Phi$. Then the corresponding function $u=\widehat{u}(t)$ is a solution of the finite jet approximation.

Proof. We are to show that if $\rho$ is strictly increasing and $\varphi=\hat{\varphi}$, then $u=\hat{u}$ satisfies conditions (A) through (D) of $\S 3$, as modified in $\S 4$. Conditions (A) and (B), as well as the continuity of $u$ for all $|t| \leqq 1$ follow from the definition of $\widehat{u}$ together with the symmetry of $D$. The restriction of $\hat{u}$ to $|t|=1$ is a homeomorphism of the unit circumference onto $C$, and consequently $\hat{v}(\sigma) e^{i \hat{\varphi}(\sigma)}$ is a homeomorphism of $[0, \pi / 2]$ onto $C_{I}$. Since $\rho$ is strictly increasing it follows from Lemma 1 that $v(\sigma) e^{i \varphi(\sigma)}$ is a similar homeomorphism. Moreover there is only one value of $\phi$ for each point on $C_{I}$, and, since $\hat{\varphi}=\varphi$, the homeomorphisms are identical. Therefore $\hat{v}=v=\left|u\left(e^{i \sigma}\right)\right|$ satisfies (7). Condition (D) is satisfied since $\Phi$ and $v$ are both strictly increasing; and the proof is complete.

It should be noticed that the strictly increasing property of $\varphi$ plays an essential role in the proof, beyond the relatively unimportant fact that it is needed for condition (D). If $\varphi$ were constant on some interval, we could not conclude that $v=\hat{v}$ there, and equation (7) would not follow.

Now the family $\Phi$ is clearly a closed, bounded, convex subset of the Banach space of continuous functions on $[0, \pi / 2]$ with the "uniform norm" 
$\|\varphi\|=\sup \{|\varphi(\sigma)|: 0 \leqq \sigma \leqq \pi / 2\}$. In the next three sections it is shown that $T$ is a continuous transformation of $\Phi$ into itself whose range has compact closure (i.e., $T$ is a "completely continuous operator"). In accordance with a theorem of Schauder [2], it follows that $T$ has a fixed point. To complete the proof of Theorem 2, it is shown in $\S 9$ that every fixed point is strictly increasing.

\section{Proof that $T$ maps $\Phi$ into itself.}

LEMma 3. Let $f(t)$ be regular on the unit disk, continuous on the closure, real on the real diameter, and pure imaginary on the imaginary diameter. Suppose further that $f$ vanishes only at $t=0$, that $f^{\prime}(0)$ is positive, and that $F(\sigma)=\left|f\left(e^{i \sigma}\right)\right|$ is of bounded variation. Then for $0<|t|<1$,

$$
\arg \{f(t) / t\}=\int_{0}^{\pi / 2} k(t, \rho) d \log F(\rho)
$$

where the kernel $k(t, \rho)=\pi^{-1} \log \left|\left(e^{2 i \rho}-t^{2}\right) /\left(e^{-2 i \rho}-t^{2}\right)\right|$ is negative for $0<\arg t<\pi / 2$.

Proof. Let $g(t)=\log [f(t) / t]$, where the branch of the logarithm is chosen so that $g(0)=\log f^{\prime}(0)$ in real. Applying Poisson's formula we have

$$
\operatorname{Re}\{g(t)\}=\operatorname{Re}\left\{(2 \pi)^{-1} \int_{-\pi}^{\pi}\left[\left(e^{i \rho}+t\right) /\left(e^{i \rho}-t\right)\right] \log F(\rho) d \rho\right\},
$$

since $\operatorname{Re}\left\{g\left(e^{i \sigma}\right)\right\}=\log F(\sigma)$. Since the integral is regular for $|t|<1$, it differs from $g(t)$ by an imaginary constant, which is zero since both functions are real at $t=0$. Integration by parts gives

$$
\arg \{f(t) / t\}=\Im_{\mathfrak{m}}\{g(t)\}=\pi^{-1} \int_{-\pi}^{\pi} \log \left|e^{i \rho}-t\right| d \log F(\rho) .
$$

This can be reduced to the required formula by means of the symmetry conditions $F(\sigma)=F(-\sigma)=F(\pi-\sigma)$. The proof is completed by observing that $\left|e^{2 i \rho}-t^{2}\right| \leqq\left|e^{-2 i \rho}-t^{2}\right|$ when $e^{i \rho}$ and $t$ are both in the first quadrant, so that $k(t, \rho) \leqq 0$ there.

Returning to the operator $T$, it follows from the definition of $\hat{u}$ that $\widehat{u}\left(e^{i \sigma}\right)=\hat{v}(\sigma) e^{i \hat{\varphi}(\sigma)}$ is a homeomorphism of $[0, \pi / 2]$ onto $C_{I}$, and in the same direction as $v(\sigma) e^{i \varphi(\sigma)}$. Hence $\hat{\varphi}$, like $\varphi$, is nondecreasing between the limits $0, \pi / 2$, and $\hat{v}$ is increasing. Taking $f(t)=\widehat{u}(t)$ in Lemma 3, so that $F(\sigma)=\hat{v}(\sigma)$, it follows that $\arg \left\{\hat{u}\left(r e^{i \sigma}\right)\right\}-\sigma \leqq 0$ for $0<r<1$, $0 \leqq \sigma \leqq \pi / 2$. In the limit $r=1$ this gives $\widehat{\phi}(\sigma) \leqq \sigma$. Thus $T \mathscr{\rho}=\widehat{\phi}$ belongs to $\Phi$. 
7. Continuity of $T$. The proof of continuity is based on the following theorem of Radó [3] ${ }^{1}$ :

THEOREM 3. Let $D$ be a domain containing the origin and bounded by a closed Jordan curve $C$. Let $u(t)$ map $|t|<1$ conformally onto $D$, subject to the conditions $u(0)=0, u^{\prime}(0)>0$. If $\left\{D_{n}\right\}$ is a sequence of such regions "approaching" $D$, then the corresponding sequence of functions $\left\{u_{n}\right\}$ approaches $u$ uniformly on $|t| \leqq 1$ if and only if the Fréchet distance between the boundaries $C_{n}$ and $C$ approaches zero. (The Fréchet distance is the infimum, over all homeomorphisms between the curves, of the maximum distance between corresponding points.)

Now let $\mathcal{P}$ and $\widetilde{\rho}$ be any two members of $\Phi$, and let $v, C_{I}, C$, and $\widetilde{v}, \widetilde{C}_{I}, \widetilde{C}$ be the functions and curves involved in the construction of $T \Phi$ and $T \tilde{\Phi}$, respectively. We will show that the Fréchet distance between $C$ and $\widetilde{C}$ is not greater than $k\|\varphi-\widetilde{\varphi}\|$, where the number $k$ is independent of $\varphi$ and $\widetilde{\varphi}$. Then it follows from Theorem 3 and the fact that $C$ is bounded away from the origin (i.e., $|u| \geqq 1$ on $C$ ) that $T$ is continuous.

Suppose that $\|\mathscr{P}-\tilde{\varphi}\|>0$; and let $\left[0, \sigma_{0}\right],\left[0, \widetilde{\sigma}_{0}\right]$ be the maximal intervals on which $\varphi, \widetilde{\Phi}$ vanish, respectively. Let $\left[0, \sigma_{1}\right]$ be the maximal interval on which $\varphi \leqq 2\|\rho-\widetilde{\phi}\|$. Clearly $\pi / 2 \geqq \sigma_{1}>\max \left\{\sigma_{0}, \widetilde{\sigma}_{0}\right\}$. Now consider the correspondence $\sigma \rightarrow \sigma^{*}$ defined by

$$
\sigma^{*}=\left\{\begin{array}{ll}
\widetilde{\sigma}_{0}+\left(\sigma-\sigma_{0}\right)\left(\sigma_{1}-\widetilde{\sigma}_{0}\right) /\left(\sigma_{1}-\sigma_{0}\right) & \text { for } \sigma_{0,}^{\digamma} \leqq \sigma \leqq \sigma_{1} \\
\sigma & \text { for } \sigma \geqq \sigma_{1}
\end{array} .\right.
$$

This is a homeomorphism of $\left[\sigma_{0}, \pi / 2\right]$ onto $\left[\widetilde{\sigma}_{0}, \pi / 2\right]$; and in view of Lemma 1 the correspndence $v(\sigma) e^{i \varphi(\sigma)} \rightarrow \widetilde{v}\left(\sigma^{*}\right) e^{\tilde{i}\left(\sigma^{*}\right)}$ is a homeomorphism of $C_{I}$ onto $\widetilde{C}_{I}$. The distance between corresponding points does not exceed

$$
\begin{aligned}
& \left|v(\sigma) e^{i \varphi(\sigma)}-v\left(\sigma^{*}\right) e^{i \varphi\left(\sigma^{*}\right)}\right|+\left|v\left(\sigma^{*}\right) e^{i \varphi\left(\sigma^{*}\right)}-\widetilde{v}\left(\sigma^{*}\right) e^{\tilde{\varphi}\left(\sigma^{*}\right)}\right| \\
& \leqq \\
& \quad+M(\sigma)-v\left(\sigma^{*}\right)|+M| \varphi(\sigma)-\varphi\left(\sigma^{*}\right)|+| v\left(\sigma^{*}\right)-\widetilde{v}\left(\sigma^{*}\right) \mid \\
& \quad+M\left|\varphi\left(\sigma^{*}\right)-\widetilde{\varphi}\left(\sigma^{*}\right)\right|
\end{aligned}
$$

where $M$ is the upper bound on $v$ in (8). The first two terms vanish except when $\sigma, \sigma^{*} \in\left[0, \sigma_{1}\right]$. If $K$ is a Lipschitz constant such that $\left|x^{1 / 3 \beta}-y^{1 / 3 \beta}\right| \leqq K|x-y|$ for $1 \leqq x \leqq y \leqq M^{3 \beta}$, it follows that

$$
\left|v(\sigma)-v\left(\sigma^{*}\right)\right| \leqq K \mid \lambda \int_{\sigma}^{\sigma^{*}} h_{\gamma}(\rho) \sin \left[\beta \varphi(\rho) d \rho \mid \leqq K M^{3 \beta} \beta \cdot 2\|\varphi-\widetilde{\varphi}\| .\right.
$$

Moreover $M\left|\varphi(\sigma)-\varphi\left(\sigma^{*}\right)\right| \leqq 4 M\|\varphi-\widetilde{\phi}\|$,

$$
\begin{aligned}
\left|v\left(\sigma^{*}\right)-\widetilde{v}\left(\sigma^{*}\right)\right| & \leqq K \lambda \int_{\sigma}^{\sigma^{*}} h_{\gamma}(\rho)|\sin \beta \varphi(\rho)-\sin \beta \widetilde{\varphi}(\rho)| d \rho \\
& \leqq K M^{3 \beta} \beta\|\varphi-\widetilde{\varphi}\|,
\end{aligned}
$$

1 See also the discussion in the opening paragraphs of [4]. 
and $M\left|\rho\left(\sigma^{*}\right)-\widetilde{\varphi}\left(\sigma^{*}\right)\right| \leqq M\|\varphi-\widetilde{\varphi}\|$. Hence the Fréchet distance between $C_{I}$ and $\widetilde{C}_{I}$ is not greater than $\left(3 \beta K M^{3 \beta}+5 M\right)\|\varphi-\widetilde{\phi}\|$; and in view of the symmetry, this is also an upper bound on the Fréchet distance between $C$ and $\widetilde{C}$.

8. Complete continuity. To establish the complete continuity of $T$ we will show that its range is an equicontinuous family, and hence has compact closure. The chief tool is ${ }^{2}$.

LEMma 4. Let $|t|<1$ be mapped conformally onto a simply connected domain $D$ of finite area $A$. Let $t_{0}$ be a point on $|t|=1$, and $k_{r}$ be that part of the circle $\left|t-t_{0}\right|=r$ which is contained in $|t|<1$. Then for every $r, 0<r<1$, there is an $s, r \leqq s \leqq r^{1 / 2}$, such that the image of $k_{s}$ is a crosscut of $D$ of length $l_{s} \leqq(-2 \pi A / \log r)^{1 / 2}$.

Now let $D$ be the domain in the $u$-plane corresponding to $\mathcal{P} \in \Phi$. The function $\hat{\varphi}=\arg \hat{u}\left(e^{i \sigma}\right)$, which is equal to $T \varphi$ on $[0, \pi / 2]$, is actually defined and monotonic for all real $\sigma$. To obtain a modulus of continuity for $\hat{\varphi}$, let $\delta_{0}=e^{-(\pi M)^{2} / 2}$ where $M$ is given by (8), and let $\sigma, \sigma^{\prime}, \delta$ be any three numbers such that $0<\left|\sigma-\sigma^{\prime}\right| \leqq \delta \leqq \delta_{0}$. Then the points $e^{i \sigma}$, $e^{i \sigma^{\prime}}$ are inside the circle of radius $r=\delta$ about $t_{0}=e^{i \sigma}$. Applying Lemma 4, let $e^{i \tau}$ and $e^{i \tau^{\prime}}$ be the endpoints of an arc $k_{s}$. Since $\hat{\varphi}$ is monotonic we have $\left|\hat{\varphi}(\sigma)-\hat{\varphi}\left(\sigma^{\prime}\right)\right| \leqq\left|\hat{\varphi}(\tau)-\hat{\varphi}\left(\tau^{\prime}\right)\right|$. Now $D$ is contained in the disk $|u| \leqq M$, so the length of $k_{s}$ is $l_{s} \leqq\left(-2 \pi^{2} M^{2} / \log \delta\right)=2\left(\log \delta_{0} / \log \delta\right) \leqq 2$. Since the boundary of $D$ is outside the unit circle, it follows that $\left|\widehat{\varphi}(\tau)-\hat{\varphi}\left(\tau^{\prime}\right)\right|=\left|\arg \hat{u}\left(e^{i \tau}\right)-\arg \hat{u}\left(e^{i \tau^{\prime}}\right)\right|$ is not greater than the angle subtended at the origin by a chord of the unit circle of length $l_{s}$. Therefore the principal value of $2 \arcsin \left(\log \delta_{0} / \log \delta\right)$ is a modulus of continuity of $\hat{\rho}$ for $\delta \leqq \delta_{0}$, which is independent of $\mathcal{P}$.

The proof that $T$ has a fixed point is now complete.

9. Strictly increasing nature of the fixed points. For the proof that every fixed point of $T$ is a strictly increasing function it is convenient to change variables from $t$ to $\zeta$ by the transformation

$$
\zeta(t)=\frac{\lambda}{2} \log \frac{B t^{2}}{\left(t^{2}+\gamma^{2}\right)\left(t^{2}+\gamma^{-2}\right)}, \quad B>0 .
$$

Taking the principal branch of the logarithm, we see that $\zeta(t)$ maps the first quadrant of the unit disk onto the strip $0 \leqq \Im \mathfrak{m}\{\zeta\} \leqq \lambda \pi / 2$ so that the real radius, the circumference, and the imaginary segment $t=i \tau$, $\gamma<\tau<1$, go into the real axis, and the rest of the imaginary radius goes into the line $\mathfrak{F} \mathfrak{m}\{\zeta\}=\lambda \pi / 2$. At the same time we change variables

${ }^{2}$ The statement of Lemma 4 follows Warschawski [5], who gives a proof and refers to J. Wolff [6]. The essential idea is given earlier by Courant [7]. 
from $\sigma$ to $\zeta\left(e^{i \sigma}\right)$, so that $\varphi, v$, and $\hat{v}$ become functions of $\zeta$, defined on the real segment $a \leqq \zeta \leqq b$ corresponding to $0 \leqq \sigma \leqq \pi / 2$. The location of this segment can be chosen at will by adjusting the value of $B$ in (9).

With these changes the relation (7) between $\mathcal{Q}$ and $v$ becomes

$$
v(\zeta)=\left[1+\int_{a}^{\zeta} \sin [\beta \mathcal{P}(\xi)] d \xi\right]^{1 / 3 \beta}
$$

since $\lambda h_{\gamma}(\sigma)=d \zeta\left(e^{i \sigma}\right) / d \sigma$; and the relation $\widehat{v}(\sigma)=\left|\widehat{u}\left(e^{i \sigma}\right)\right|$ becomes $\hat{v}(\zeta)=$ $|\widehat{u}(\zeta)|, a \leqq \zeta \leqq b$.

Let $\phi(\zeta)$ correspond in this way to a fixed point of $T$. In accordance with Lemma 1 and the construction of $\widehat{u}$, both $v e^{i \varphi}$ and $\hat{v} e^{i \varphi}$ are homeomorphisms of $[a, b]$ onto $C_{I}$; where $v$ and $\widetilde{v}$ are both strictly increasing. Since $C_{I}$ intersects the real and imaginary axes only in its endpoints we have

1. $\mathscr{P}(\zeta)$ is strictly increasing at the endpoints $a, b$.

Now let $\mathscr{C}$ be the open real set consisting of all $\zeta \in[a, b]$ such that $\mathcal{P}$ is constant on some neighborhood of $\zeta$; and let $\mathscr{T}$ be the complement of $\mathscr{C}$ relative to $[a, b]$.

2. For all $\zeta \in \mathscr{T}, v(\zeta)=\widehat{v}(\zeta)$.

Proof. We will show that $v(\zeta) \neq \hat{v}(\zeta)$ implies $\zeta \in \mathscr{C}$. If $v(\zeta)>\hat{v}(\zeta)$, there is a $\zeta_{1}<\zeta$ such that $v\left(\zeta_{1}\right)=\hat{v}(\zeta)$ and a $\zeta_{2}>\zeta$ such that $v(\zeta)=\hat{v}\left(\zeta_{2}\right)$. Since there is at most one point on $C_{I}$ for each value of $|u|$, we have $v\left(\zeta_{1}\right) e^{i \varphi\left(\zeta_{1}\right)}=\hat{v}(\zeta) e^{i \varphi(\zeta)}$ and $v(\zeta) e^{i \varphi(\zeta)}=\hat{v}\left(\zeta_{2}\right) e^{i \varphi\left(\zeta_{2}\right)}$. Hence $\varphi\left(\zeta_{1}\right)=\phi\left(\zeta_{2}\right)$, so that $\Phi$ is constant on the interval $\left(\zeta_{1}, \zeta_{2}\right)$, and $\zeta \in \mathscr{C}$. Similarly, $v(\zeta)<\hat{v}(\zeta)$ implies $\zeta \in \mathscr{R}$.

3. The function $\hat{u}(\zeta)$ is regular on the real set $\mathscr{C}$. Moreover the logarithmic derivative $\hat{u}^{\prime} \mid \widehat{u}=\hat{v}^{\prime} / \hat{v}$ is strictly increasing on each open interval contained in $\mathscr{C}$.

The proof depends on the following result, which is essentially a corollary of a Lavrentieff-Serrin comparison theorem [8]:

Lemma 5. Let $R_{1}$ and $R_{2}$ be two closed simply-connected regions such that $R_{1}$ contains $R_{2}$. Suppose that there are continuous functions $f_{1}(\zeta)$ and $f_{2}(\zeta)$ which map the strip $0 \leqq \Im m\{\zeta\} \leqq Q$ onto $R_{1}$ and $R_{2}$, respectively, and which are conformal on the interior of the strip. Let the image of $\Im \mathfrak{m}\{\zeta\}=Q$ under $f_{2}$ be a subarc of the corresponding image under $f_{1}$; and let the images of $\mathfrak{I m}\{\zeta\}=0$ have a common point $f_{1}\left(\zeta_{1}\right)=$ $f_{2}\left(\zeta_{2}\right)$. If $f_{1}$ has a continuous nonvanishing derivative $f_{1}^{\prime}$ on a semineighborhood $\left|\zeta-\zeta_{1}\right|<\rho$, $\Im \mathfrak{m}\{\zeta\} \geqq 0$, of $\zeta_{1}$ and $f_{2}$ has a continuous 
nonvanishing derivative $f_{2}^{\prime}$ on a similar semi-neighborhood of $\zeta_{2}$, then $\left|f_{1}^{\prime}\left(\zeta_{1}\right)\right| \leqq\left|f_{2}^{\prime}\left(\zeta_{2}\right)\right|$. Equality holds only if $R_{1}$ and $R_{2}$ are identical.

To prove 3, we note first that on any open interval $(\alpha, \beta)$ contained in $\mathscr{C}, e^{-\hat{\varphi}(\alpha)} \hat{u}(\zeta)=\hat{v}(\zeta)$ is real. Hence, by the reflection principle, $\hat{u}$ is regular there. Moreover its derivative does not vanish since $\hat{u}$ is univalent.

Let $\zeta_{1}, \zeta_{2}$ be points in $(\alpha, \beta)$ such that $\zeta_{1}<\zeta_{2}$; and let $f_{1}(\zeta)=\widehat{u}(\zeta)$, $f_{2}(\zeta)=\mu \widehat{u}(\zeta)$, where $\mu=\widehat{u}\left(\zeta_{1}\right) / \widehat{u}\left(\zeta_{2}\right)<1$. Since $\phi=\arg \hat{u}$ is increasing on $[a, b], f_{1}$ and $f_{2}$ map $0 \leqq \Im \mathfrak{m}\{\zeta\} \leqq \lambda \pi / 2$ onto starlike regions $R_{1}, R_{2}$ of the first quadrant such that $R_{1}$ contains $R_{2}$. Moreover $f_{1}$ maps $\Im \mathfrak{m}\{\zeta\}=$ $\lambda \pi / 2$ (corresponding to the imaginary segment $0<t / i<\gamma$ in the $t$-plane) onto an imaginary segment $0<u / i<k$, and the corresponding image under $f_{2}$ is the subsegment $0<u / i<\mu k$. Applying Lemma 5 we obtain $\left|\hat{u}^{\prime}\left(\zeta_{1}\right)\right|<\left|\mu \hat{u}^{\prime}\left(\zeta_{2}\right)\right|$. Proposition 3 follows since $\hat{u}=e^{i \varphi(\alpha)} \hat{v}, \hat{u}^{\prime}=$ $e^{i \varphi(\alpha)} \hat{v}^{\prime}$ on $(\alpha, \beta)$, where $\hat{v}^{\prime}>0$.

4. For all $\zeta \in \mathscr{C}, \hat{v}(\zeta)<v(\zeta)$. Moreover, if $(\alpha, \beta)$ is any maximal open interval contained in $\mathscr{C}, \lim _{\zeta \rightarrow \alpha_{+}} \hat{v}^{\prime}(\zeta) / \hat{v}(\zeta)<v^{\prime}(\alpha) / v(\alpha)$.

Proof. If $(\alpha, \beta)$ is a maximal open interval contained in $\mathscr{C}$, then its endpoints belong to $\mathscr{T}$. In view of 2 and $3, \log \hat{v}$ is a strictly "concave" function on $(\alpha, \beta)$, varying between the limits $\log v(\alpha), \log v(\beta)$. According to (7), on the other hand, $\log v(\zeta)$ varies linearly there since $\varphi$ is constant; and the proposition follows.

Now we restrict our attention to a fixed maximal open interval $(\alpha, \beta)$ of $\mathscr{C}$, and adjust the parameter $B$ in (9) so that $\alpha=0$. Let $\Delta$ denote a neighborhood $|\zeta|<R$ of the origin, cut along the real segment $-R<\zeta<0$. In view of $1, \alpha$ is greater than $a$, and we can choose $R>0$ so that the segment $-R<\zeta<0$ is contained in $[a, b]$, the segment $0<\zeta<R$ is contained in $(\alpha, \beta)$, and $R<\lambda \pi / 2$. Finally we set $g(\zeta)=\log \widehat{u}(\zeta)$, where $\Im \mathfrak{m}\{g(\zeta)\}=\phi(0)$ for $0 \leqq \zeta \leqq R$.

5. For all $\zeta \in \Delta$ we have

$$
g(\zeta)=p(\zeta)-\pi^{-1} \int_{-R}^{0} \log (\zeta-\tau) d \varphi(\tau)
$$

where $p(\zeta)$ is a convergent power series in $\zeta$, and the branch of the logarithm is such that $\log (\zeta-\tau)$ is real for $\zeta-\tau>0$.

Proof. Since $g(\zeta)-i \varphi(0)$ is real for $0 \leqq \zeta \leqq R$, the analytic continuation of $g$ across this segment is given by $g(\zeta)=2 i \phi(0)+\overline{g(\bar{\zeta})}$, so that $g$ is continuous on the cut disk $\Delta$. The discontinuity across the cut is $g(\zeta)-g(\bar{\zeta})=2 i[\varphi(0)-\phi(\zeta)]$ for $\arg \zeta=-\pi$, arg $\bar{\zeta}=\pi$.

Now the integral in (11) is defined and regular except on the cut, 
so this equation serves to define $p(\zeta)$ at least for $-\pi<\arg \zeta<\pi$. Let $\zeta=\xi+i \eta, \eta<0$, and consider the difference $p(\zeta)-p(\bar{\zeta})=g(\zeta)-g(\bar{\zeta})+$ $\pi^{-1} \int_{-R}^{0} \log [(\zeta-\tau) /(\bar{\zeta}-\tau)] d \varphi(\tau)$. Here $\log [(\zeta-\tau) /(\bar{\zeta}-\tau)]=2 i \arg (\zeta-\tau)$ is bounded, and as $\eta \rightarrow 0$ it approaches $-2 \pi i$ for $\xi<\tau$ and 0 for $\xi>\tau$. Therefore $p(\zeta)-p(\bar{\zeta})$ is bounded and

$$
\lim _{\eta \rightarrow 0}[p(\xi+i \eta)-p(\xi-i \eta)]=2 i[\varphi(0)-\varphi(\xi)]-2 i \int_{\xi}^{0} d \varphi(\tau)=0 .
$$

To show that $p$ is regular for all $|\zeta|<R$, let $A$ be the circular arc $|\zeta|=R, \mathfrak{F}\{\zeta\} \geqq \varepsilon$, where $0<\varepsilon<R$, and let $B$ be the segment $\Im \mathfrak{m}\{\zeta\}=\varepsilon$ joining the endpoints of $A$. Let $\Gamma$ be the contour $A+B$, and $\bar{\Gamma}$ be its reflection across the real axis. Now take $\zeta_{1}$ inside either $\Gamma$ or $\bar{\Gamma}$, and consider the sum of the integrals of $p(\zeta) /\left(\zeta-\zeta_{1}\right)$ around $\Gamma$ and $\bar{\Gamma}$. The total contribution of the horizontal segments is

$$
\begin{aligned}
& \int_{B}\left[\frac{p(\zeta)}{\zeta-\zeta_{1}}-\frac{p(\bar{\zeta})}{\bar{\zeta}-\zeta_{1}}\right] d \zeta \\
& \quad=\int \frac{[p(\xi+i \varepsilon)-p(\xi-i \varepsilon)]\left(\xi-\zeta_{1}\right)-i \varepsilon[p(\xi+i \varepsilon)+p(\xi-i \varepsilon)]}{\left(\xi+i \varepsilon-\zeta_{1}\right)\left(\xi-i \varepsilon-\zeta_{1}\right)} d \xi .
\end{aligned}
$$

In view of (11) and (12) this vanishes as $\varepsilon \rightarrow 0$, so that $2 \pi i p\left(\zeta_{1}\right)=$ $\int_{|\zeta|=R} p(\zeta)\left(\zeta-\zeta_{1}\right)^{-1} d \zeta$. Thus $p(\zeta)$ is regular and has a convergent Maclauren series for $|\zeta|<R$. Finally, (11) holds on the cut, since $g(\zeta)-p(\zeta)$ is continuous on each side and the unbounded part of the integrand, $\log |\zeta-t|$, converges monotonically as $\mathfrak{I} \mathfrak{m}\{\zeta\}$ vanishes.

6. The fixed point $\varphi$ is strictly increasing.

Proof. We will use the representation (11) for $g=\log \hat{u}$ to obtain

$$
\lim _{\zeta \rightarrow 0_{+}} \hat{v}^{\prime}(\zeta) / \hat{v}(\zeta) \geqq v^{\prime}(0) / v(0) .
$$

This contradicts 4 if the open set $\mathscr{C}$ contains an open interval. Therefore $\mathscr{C}$ is void and $\varphi$ is strictly increasing.

For $0<\zeta<R$ we have $g^{\prime}(\zeta)=\hat{v}^{\prime}(\zeta) / \hat{v}(\zeta)=p^{\prime}(\zeta)-\pi^{-1} \int_{-R}^{0}(\zeta-\tau)^{-1} d \varphi(\tau)$. Here $g^{\prime}$ is positive, so the last term, being negative, converges monotonically to a finite limit. Hence

$$
\lim _{\zeta \rightarrow 0+} \hat{v}^{\prime}(\zeta) / \hat{v}(\zeta)=p^{\prime}(0)+\pi^{-1} \int_{-R}^{0} \tau^{-1} d \varphi(\tau) .
$$

For $\arg \zeta=\pi,-R<\zeta<0$, we have

$$
g(0)^{3}-g(\zeta)=p(0)-p(\zeta)+\pi^{-1} \int_{-R}^{0} \log (1-\zeta / \tau) d \varphi(\tau) .
$$


The last term is

$$
\pi^{-1} \int_{-R}^{0} \log |1-\zeta| \tau \mid d \varphi(\tau)+i[\varphi(0)-\varphi(\zeta)]
$$

Taking real parts and using the inequality $\log x \leqq x-1$ we obtain $\log \hat{v}(0)-\log \hat{v}(\zeta) \leqq p(0)-p(\zeta)-\pi^{-1} \int_{-R}^{\zeta}(\zeta / \tau) d \varphi(\tau)+\pi^{-1} \int_{\zeta}^{0}(\zeta / \tau) d \varphi(\tau)$. In view of 2 and $4, \widehat{v}(0)=v(0)$ and $\widehat{v}(\zeta) \stackrel{-R}{\leqq} v(\zeta)$, so that

$[\log v(0)-\log v(\zeta)] /(-\zeta)$

$$
\leqq[p(0)-p(\zeta)] /(-\zeta)+\pi^{-1} \int_{-R}^{\zeta} \tau^{-1} d \varphi(\tau)-\pi^{-1} \int_{\zeta}^{0} \tau^{-1} d \varphi(\tau)
$$

Taking the limit $\zeta \rightarrow 0 \ldots$ and combining with (14) gives (13).

The proof of Theorem 2 is now complete.

10. Analytic continuation of finite jet solutions. To obtain the infinite free boundary solutions as limits of finite jet solutions we require

LeMma 6. For each pair $\lambda, \beta$ such that $\lambda>0,0<\beta<2$, there is a domain $\Delta$ in the t-plane containing the closed unit disk except for $t= \pm 1, \pm i$, such that for all $0<\gamma<1$ the finite jet solutions can be continued analytically onto $\Delta$, where they form a locally uniformly bounded family of regular functions.

The fact that analytic continuation beyond the unit disk is possible follows from the work of Lewy [9]. To establish the required uniform estimates we will give a separate proof, based on the differential equation

$$
d \widetilde{v} / d t=f(t, \widetilde{v}) \equiv t \zeta^{\prime}(t)\left[(u / \widetilde{v})^{\beta}-(\widetilde{v} / u)^{\beta}\right] /\left[6 \beta \widetilde{v}^{3 \beta-1}\right]
$$

where $\zeta(t)$ is given by (9). This is obtained from the boundary condition (7) by setting $t=e^{i \sigma}, \widetilde{v}(t)=v(\sigma)$, differentiating, and observing that $\lambda h_{\gamma}(\sigma)=i e^{i \sigma} \zeta^{\prime}\left(e^{i \sigma}\right)$ and $e^{i \beta \varphi}=(u / \widetilde{v})^{\beta}$. The $\operatorname{method}^{3}$ is to show that equation (15) has a solution $\widetilde{v}$ which is regular on a domain of the form $t=r e^{i \sigma}, R(\sigma)<r<1,0<\sigma<\pi / 2$, continuous and nonvanishing on the closure of this domain, and equal to $v(\sigma)$ for $t=e^{i \sigma}$. Then $\widetilde{\phi}=-i \log u / \widetilde{v}$ is regular on the same domain, continuous on the closure, and equal to $\varphi(\sigma)$ for $t=e^{i \sigma}$. Since both $\widetilde{v}$ and $\widetilde{\varphi}$ are equal on the circumference, they can be continued across this circular arc by reflection; and the required analytic continuation of $u$ in the first quadrant is given by $u=\tilde{v} e^{i \tilde{\varphi}}$ for $t=r e^{i \sigma}, 1<r<1 / R(\sigma)$. The continuation in the other quadrants is given by the symmetry of $u$. Lemma 6 is valid provided [11].

${ }^{3}$ Suggested by the work of Vitousek [10], and illustrated for a closely related case in 
$R(\sigma)$, together with local bounds on $|u|$, can be chosen independently of $\gamma$. To this end we have

1. The solutions $u(t)$ satisfy $|t| \leqq|u(t)| \leqq[1-\lambda \log \cos \theta]^{1 / 3 \beta}$ on each sector $|t| \leqq 1,0 \leqq \arg t \leqq \theta<\pi / 2$.

Proof. The first inequality follows by applying the maximum principle to $t / u(t)$, which is regular for $|t|<1$ and whose maximum absolute value for $|t|=1$ is $1 /|u(1)|=1$.

Since $u$ maps $|t|<1$ onto a starlike domain we have (see [12], Ch. V) $\operatorname{Re}\left\{t u^{\prime} \mid u\right\}=\partial \log |u| / \partial \log |t| \geqq 0$ for $|t|<1$, so that $|u|$ is increasing on each radius. Since $\left|u\left(e^{i \sigma}\right)\right|$ is also increasing for $0 \leqq \sigma \leqq \pi / 2$, it follows that $|u(t)| \leqq v(\theta)$ on the sector in question. Estimating $v(\theta)$ with the help of (6) and (7) yields the second inequality, since $0 \leqq h_{\gamma}(\sigma) \leqq$ $\tan \sigma$.

The next proposition follows easily with the help of 1:

2. For $0<\theta<\pi / 2$, let $\Delta_{\theta}$ be the domain $\frac{1}{2}<|t|<1,0<\arg t<\theta$, and let $D_{\theta}$ be the annulus $\frac{1}{2} \leqq|\widetilde{v}| \leqq \frac{1}{2}+[1-\lambda \log \cos \theta]^{1 / 3 \beta}$. Then the function $f(t, \widetilde{v})$ of equation (15) is regular in both arguments on the product region $\Delta_{\theta} \times D_{\theta}$, and continuous on the closure $\bar{\Delta}_{\theta} \times D_{\theta}$. Moreover there are numbers $M_{\theta}$ and $L_{\theta}$, independent of $\gamma$, such that for $t \in \bar{\Delta}_{\theta}$ and $\widetilde{v}, \widetilde{v}_{1} \in D_{\theta},|f(t, \widetilde{v})| \leqq M_{\theta}$ and $\left|f(t, \widetilde{v})-f\left(t, \widetilde{v}_{1}\right)\right| \leqq L_{\theta}\left|\widetilde{v}-\widetilde{v}_{1}\right|$.

To construct the required solution of (15) near $t_{0}=e^{i \theta}$, let $K_{\theta}$ be the intersection of $\bar{J}_{\theta}$ with the disk $\left|t-t_{0}\right| \leqq \min \left\{1 / 2 M_{\theta}, 1 / 2 L_{\theta}, 1 / 2\right\}$, and let $V$ be the family of functions $\widetilde{v}(t)$ which map $K_{\theta}$ continuously into $D_{\theta}$ and are regular on the interior of $K_{\theta}$. Then $V$ is a complete space with respect to the uniform metric. Now consider the operator $J$ on $V$ defined by $J \widetilde{v}=v(\theta)+\int_{t_{0}}^{t} f(s, \widetilde{v}(s)) d s$, where the path of integration is in $K_{\theta}$. It follows from 1,2 , and the definition of $K_{\theta}$, that $J$ maps $V$ into itself and is a contraction operator. Therefore $J$ has a unique fixed point $\widetilde{v}_{0}$, which is a solution of (15) and equal to $v(\theta)=\left|u\left(t_{0}\right)\right|$ at $t_{0}$. Since (15) was chosen so that $|u(t)|$ is a solution on the arc $|t|=1$, it follows from the contraction property of $J$ that $\widetilde{v}_{0}=|u|$ there. Also because of the contraction property, solutions corresponding to two different values of $\theta$ are equal on the intersection of the corresponding regions $K_{\theta}$. This provides a solution $\widetilde{v}$ on the region $t=r e^{i \sigma}, R(\sigma)<r \leqq 1$, $0<\sigma<\pi / 2$, with $R(\sigma)=1-\min \left\{1 / 2 M_{\sigma}, 1 / 2 L_{\sigma}, 1 / 2\right]$, such that $\left|\widetilde{v}\left(r e^{i \sigma}\right)\right| \leqq$ $1 / 2+[1-\lambda \log \cos \sigma]^{1 / 3 \beta}$ and $|u| \geqq 1 / 2$. Lemma 6 follows in view of the remarks preceding 1 .

\section{Existence of infinite jet solutions.}

THeorem 4. For every pair $\lambda, \beta$ such that $\lambda>0,0<\beta<2$, the 
infinite free boundary problem described in $\S 3$ has a solution.

Proof. It follows from Lemma 6, in accordance with Montel's Theorem, that the family of all finite jet solutions for given $\lambda, \beta$, is normal on a domain $\Delta$ containing the closed unit disk, with $t= \pm 1, \pm i$ excluded. Hence there is a sequence $\left\{u_{n}\right\}$ of such functions for which the corresponding sequence $\left\{\lambda_{n}\right\}$ approaches 1 , and which converges uniformly on every closed subset of $\Delta$. We will show that the limit, $u$, of this sequence satisfies conditions (A) through (D) of $\S 3$.

First, $u$ is clearly regular, not only for $|t|<1$, but also for $|t|=1$, $t \neq \pm 1, \pm i$. To show that $u$ is continuous for $|t| \leqq 1, t \neq \pm i$, it is sufficient, in view of symmetry, to establish continuity in the first quadrant at $t=1$, where $u(1)=\lim u_{n}(1)=1$. According to Proposition 1 of $\S 9$ and the last paragraph of $\S 6$, each $u_{n}$ satisfies $r \leqq\left|u_{n}\left(r e^{i \sigma}\right)\right| \leqq$ $[1-\lambda \log \cos \sigma]^{1 / 3 \beta}$ and $0 \leqq \arg u_{n}\left(r e^{i \sigma}\right) \leqq \sigma$ in the first quadrant. Therefore $u$ satisfies the same estimates, and the continuity follows. Since each $u_{n}$ is univalent for $|t|<1, u$ is either univalent or constant (see [12], Ch. IV); and the latter case is ruled out because $u(1)=1$ and $u(0)=\lim u_{n}(0)=0$. Thus $u$ satisfies condition (A).

Next, $u$ satisfies condition (B) along with each $u_{n}$; and condition (C) follows from equation (7) for the $u_{n}$, since $h_{\gamma_{n}}(\sigma)$ approaches $\tan \sigma$.

Now $\varphi(\sigma)$, like each $\varphi_{n}(\sigma)=\arg u_{n}\left(e^{i \sigma}\right)$, is increasing, and $\varphi(0)=0$. Since $u$ is regular for $t=e^{i \sigma}, 0<\sigma<\pi / 2, \mathcal{D}$ is either strictly increasing or identically zero. In the latter case (2) implies $v(\sigma) \equiv 1$, so that $u \equiv 1$, which contradicts univalence. Therefore $\varphi$ and $v$ are both strictly increasing, and $v$ is unbounded near $\sigma=\pi / 2$.

It remains to show that $\varphi$ approaches $\pi / 2$ near $\sigma=\pi / 2$. Applying Lemma 3 to $u_{n}(t)$ gives $\arg \left\{u_{n}(t) / t\right\}=(1 / 3 \beta) \int_{0}^{\pi / 2} k(t, \rho) d \log v_{n}^{3 \beta}(\rho)$. Choosing an arbitrary number $\tau, 0<\tau<\pi / 2$, we have $d \log v_{n}^{3 \beta}(\rho)=\left[\lambda h_{\gamma_{n}} v_{n}^{-3 \beta} \sin \beta \varphi\right] d \rho \leqq$ $\lambda v_{n}^{-3 \beta}(\tau) h_{\gamma_{n}} d \rho$ for $\rho \geqq \tau$, and $d \log v_{n}^{3 \beta}(\rho) \leqq \lambda \tan \rho d \rho$ for all $\rho$. When $t$ is in the first quadrant, where $k(t, \rho)$ is negative, it follows that

$$
0 \geqq \arg \left\{u_{n}(t) / t\right\} \geqq(\lambda / 3 \beta) \int_{0}^{\tau} k(t, \rho) \tan \rho d \rho+\left[\lambda / 3 \beta v^{3 \beta}(\tau)\right] \int_{0}^{\pi / 2} k(t, \rho) h_{\gamma_{n}}(\rho) d \rho .
$$

The last integral can be evaluated by applying Lemma 3 to $f(t)=$ $t /\left(1+\gamma^{2} t^{2}\right)$, which satisfies $d \log \left|f\left(e^{i \rho}\right)\right|=h_{\gamma}(\rho) d \rho$; so that $\int_{0}^{\pi / 2} k(t, \rho) h_{\gamma}(\rho) d \rho=$ $-\arg \left(1+\gamma^{2} t^{2}\right)$. Taking the limit $t \rightarrow e^{i \sigma}, \tau<\sigma<\pi / 2$, and then $n \rightarrow \infty$, gives $0 \geqq \varphi(\sigma)-\sigma \geqq(\lambda / 3 \beta) \int_{0}^{\tau} k\left(e^{i \sigma}, \rho\right) \tan \rho d \rho-\left[\lambda / 3 \beta v^{3}(\tau)\right] \arg \left(1+e^{2 i \sigma}\right)$. In the limit $\sigma \rightarrow \pi / 2$ the first integral vanishes, since $k(i, \rho)=$ $\pi^{-1} \log \left|\left(e^{2 i \rho}+1\right) /\left(e^{-2 i \rho}+1\right)\right|=0$, so that $0 \geqq \varphi(\pi / 2)-\pi / 2 \geqq-\pi \lambda / 6 \beta v^{3}(\tau)$. Since $v$ is unbounded we have finally $\varphi(\pi / 2)=\pi / 2$; and the proof is complete. 


\section{REFERENCES}

1. Garrett Birkhoff and E. H. Zarantonello, Jets, wakes, and cavities, Academic Press, New York (1957), Ch. VIII, \&\& 10-11.

2. Jules Schauder, Der Fixpunktsatz in Funktionalräumen, Studia Mathematica 2 (1930), 171-180.

3. T. Radó, Sur la représentation conforme des domaines variables, Acta Litterarum ac Scientiarum, Sectio Scientiarum Mathematicarum, Universitatis Francisco-Josephinae 1 (1923), 180-186.

4. S. E. Warschawski, On conformal mapping of variable regions, U.S. Department of Commerce, National Bureau of Standards, Applied Mathematics Series, 18 (1952), 175-187.

5. S. E. Warschawski, On the degree of variation in conformal mapping of variable regions, Trans. Amer. Math. Soc., 69 (1950), 335-356.

6. J. Wolff, Sur la représentation conforme des bandes, Compositio Math., 1 (1934), 217222.

7. Hurwitz-Courant, Funktionentheorie, Springer Berlin, Dritten Auflage (1929), Kap. 6, \$ 4. 8. James Serrin, On plane and axially symmetric free boundary problems, Jour. Math. and Mech., 2 (1953), 563-575 (Appendix).

9. Hans Lewy, $A$ note on harmonic functions and a hydrodynamical application, Proc. Amer. Math. Soc., 3 (1952), 111-113.

10. Martin J. Vitousek, Some flows in a gravity field satisfying the exact free surface condition, Technical Report No. 25, Applied Mathematics and Statistics Laboratory, Stanford University, (1954).

11. D. S. Carter, Local behavior of plane gravity flows at the confluence of free boundaries and analytic fixed boundaries, Jour. Math. and Mech., 10 (1961), 441-450.

12. Zeev Nehari, Conformal Mapping, McGraw-Hill, New York, (1953).

UNiversity of CALIFORNIA, BERKELEY 



\title{
PACIFIC JOURNAL OF MATHEMATICS
}

\section{EDITORS}

\author{
Ralph S. Phillips \\ Stanford University \\ Stanford, California \\ F. H. BrownelL \\ University of Washington \\ Seattle 5 , Washington
}

A. L. Whiteman

University of Southern California

Los Angeles 7. California

L. J. PAIGE

University of California

Los Angeles 24, California

\section{ASSOCIATE EDITORS}

\author{
E. F. BECKENBACH \\ T. M. CHERRY
} \\ D. DERRY \\ M. OHTSUKA \\ UNIVERSITY OF BRITISH COLUMBIA \\ CALIFORNIA INSTITUTE OF TECHNOLOGY \\ UNIVERSITY OF CALIFORNIA \\ MONTANA STATE UNIVERSITY \\ UNIVERSITY OF NEVADA \\ NEW MEXICO STATE UNIVERSITY \\ OREGON STATE COLLEGE \\ UNIVERSITY OF OREGON \\ OSAKA UNIVERSITY \\ UNIVERSITY OF SOUTHERN CALIFORNIA
}

H. L. ROYDEN

E. SPANIER
E. G. STRAUS

F. WOLF

\section{SUPPORTING INSTITUTIONS}

\author{
STANFORD UNIVERSITY \\ UNIVERSITY OF TOKYO \\ UNIVERSITY OF UTAH \\ WASHINGTON STATE COLLEGE \\ UNIVERSITY OF WASHINGTON \\ AMERICAN MATHEMATICAL SOCIETY \\ CALIFORNIA RESEARCH CORPORATION \\ HUGHES AIRCRAFT COMPANY \\ SPACE TECHNOLOGY LABORATORIES \\ NAVAL ORDNANCE TEST STATION
}

Mathematical papers intended for publication in the Pacific Journal of Mathematics should be typewritten (double spaced), and the author should keep a complete copy. Manuscripts may be sent to any one of the four editors. All other communications to the editors should be addressed to the managing editor, L. J. Paige at the University of California, Los Angeles 24, California.

50 reprints per author of each article are furnished free of charge; additional copies may be obtained at cost in multiples of 50 .

The Pacific Journal of Mathematics is published quarterly, in March, June, September, and December. The price per volume (4 numbers) is $\$ 12.00$; single issues, $\$ 3.50$. Back numbers are available. Special price to individual faculty members of supporting institutions and to individual members of the American Mathematical Society: $\$ 4.00$ per volume; single issues, $\$ 1.25$.

Subscriptions, orders for back numbers, and changes of address should be sent to Pacific Journal of Mathematics, 103 Highland Boulevard, Berkeley 8, California.

Printed at Kokusai Bunken Insatsusha (International Academic Printing Co., Ltd.), No. 6 , 2-chome, Fujimi-cho, Chiyoda-ku, Tokyo, Japan.

PUBLISHED BY PACIFIC JOURNAL OF MATHEMATICS, A NON-PROFIT CORPORATION

The Supporting Institutions listed above contribute to the cost of publication of this Journal, but they are not owners or publishers and have no responsibility for its content or policies. 


\section{Pacific Journal of Mathematics}

\section{Vol. 11, No. $3 \quad$ BadMonth, 1961}

Errett Albert Bishop, A generalization of the Stone-Weierstrass theorem ..........

Hugh D. Brunk, Best fit to a random variable by a random variable measurable with

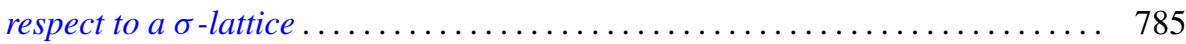

D. S. Carter, Existence of a class of steady plane gravity flows . . . . . . . . . . 803

Frank Sydney Cater, On the theory of spatial invariants ............... 821

S. Chowla, Marguerite Elizabeth Dunton and Donald John Lewis, Linear

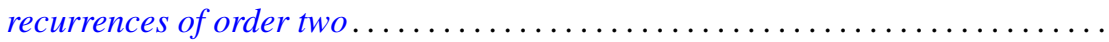

Paul Civin and Bertram Yood, The second conjugate space of a Banach algebra as

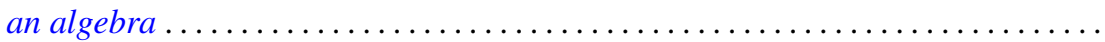

William J. Coles, Wirtinger-type integral inequalities .

Shaul Foguel, Strongly continuous Markov processes ....................

David James Foulis, Conditions for the modularity of an orthomodular lattice ...... Jerzy Górski, The Sochocki-Plemelj formula for the functions of two complex variables.

John Walker Gray, Extensions of sheaves of associative algebras by non-trivial

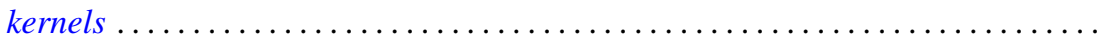

Maurice Hanan, Oscillation criteria for third-order linear differential equations .... 919 Haim Hanani and Marian Reichaw-Reichbach, Some characterizations of a class of unavoidable compact sets in the game of Banach and Mazur .............

John Grover Harvey, III, Complete holomorphs . . . . . . . . . . . . . . . . 961 Joseph Hersch, Physical interpretation and strengthing of M. Protter's method for vibrating nonhomogeneous membranes; its analogue for Schrödinger's

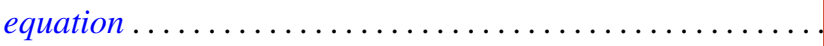

James Grady Horne, Jr., Real commutative semigroups on the plane...

Nai-Chao Hsu, The group of automorphisms of the holomorph of a group...

F. Burton Jones, The cyclic connectivity of plane continua

John Arnold Kalman, Continuity and convexity of projections and barycentric

coordinates in convex polyhedra

Samuel Karlin, Frank Proschan and Richard Eugene Barlow, Moment inequalities of

Pólya frequency functions .

Tilla Weinstein, Imbedding compact Riemann surfaces in 3-space. .

Azriel Lévy and Robert Lawson Vaught, Principles of partial reflection in the set

theories of Zermelo and Ackermann

Donald John Lewis, Two classes of Diophantine equations .....

Daniel C. Lewis, Reversible transformations . . .

Gerald Otis Losey and Hans Schneider, Group membership in rings and

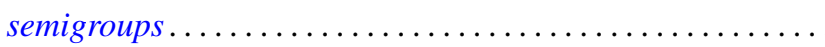

M. N. Mikhail and M. Nassif, On the difference and sum of basic sets of

polynomials.

Alex I. Rosenberg and Daniel Zelinsky, Automorphisms of separable algebras .

Robert Steinberg, Automorphisms of classical Lie algebras .... 